\title{
ESTRATÉGIAS DE COMUNICAÇÃO UTILIZADA POR INDIVÍDUOS COM NEUROPATIA/DESSINCRONIA AUDITIVA
}

\author{
Communication strategies used by people \\ with auditory neuropathy/dys-synchrony
}

\author{
Jerusa Roberta Massola de Oliveira ${ }^{(1)}$, Jéssica Kuchar ${ }^{(2)}$, Talissa Palloni Valarelli ${ }^{(3)}$
}

\section{RESUMO}

Objetivo: investigar quais são as estratégias de comunicação, segundo a natureza conforme a classificação de Boèchat (1992) utilizada por indivíduos deficientes auditivos com neuropatia/dessincronia auditiva. Métodos: para verificar a utilização ou não das estratégias de comunicação e quais as de maior e menor ocorrência foi aplicado nos indivíduos portadores de neuropatia/dessincronia auditiva o questionário elaborado por Boèchat em 1992, o qual pontua as estratégias de comunicação segundo a classificação de sua natureza. Resultados: os resultados revelam que todos os participantes do estudo recorrem ao uso das estratégias de comunicação como forma de amenizar o déficit auditivo, sendo que todas as estratégias são utilizadas. As estratégias de comunicação de natureza cognitiva são as mais utilizadas, enquanto que as desistivas são as menos utilizadas por indivíduos com neuropatia/dessincronia auditiva usuários ou não de dispositivo de amplificação sonora. Conclusão: conclui-se que o emprego das estratégias de comunicação é importante para todos os indivíduos com neuropatia/dessincronia auditiva como alternativa para facilitar a comunicação.

DESCRITORES: Estratégias; Audição; Perda Auditiva; Adulto

\section{INTRODUÇÃO}

Considerando que a audição é uma função sensorial relevante para a comunicação dos indivíduos, na medida em que promove sua integração na sociedade, qualquer desordem trará inúmeros problemas e desafios. Um exemplo,é o comprometimento da habilidade de compreensão de fala, condição presente na neuropatia/dessincronia auditiva.

O "espectro da neuropatia auditiva" é determinado pela perda da sincronia na condução nervosa,

(1) Fonoaudióloga; Divisão de Saúde Auditiva pelo Hospital de Reabilitação de Anomalias Craniofaciais da Universidade de São Paulo DAS-HRAC-USP, Bauru, SP; Doutora em Ciências da Reabilitação.

(2) Fonoaudióloga; Divisão de Saúde Auditiva pelo Hospital de Reabilitação de Anomalias Craniofaciais da Universidade de São Paulo DAS-HRAC-USP, Bauru, SP; Especialista em Audiologia Clínica e Educacional.

(3) Fonoaudióloga; Divisão de Saúde Auditiva pelo Hospital de Reabilitação de Anomalias Craniofaciais da Universidade de São Paulo DAS-HRAC-USP, Bauru, SP; Especialista em Audiologia Clínica e Educacional.

Conflito de interesses: inexistente não sendo definido o local específico da lesão, mas que pode acometer as células ciliadas internas, as sinapses entre as células ciliadas internas e $08^{\circ}$ par de nervos auditivo, o próprio nervo auditivo ou várias estruturas ${ }^{1}$. Pesquisadores alertaram que nomenclatura correta para a neuropatia/dessincronia auditiva é "espectro da neuropatia auditiva" 2.

Zeng e Liu salientaram que a neuropatia auditiva (NA) é a única alteração auditiva que apresenta consequências perceptuais diferenciadas das dificuldades observadas na perda sensorial ${ }^{3}$.

As etiologias da neuropatia/dessincronia auditiva são: prematuridade, mutações genéticas e causas infecciosas ${ }^{2}$. Alguns pesquisadores acrescentam a neuropatia sensório motora hereditária e outras neuropatias periféricas como etiologia da neuropatia/dessincronia auditiva ${ }^{4}$. Fávero e colaboradores relatam fortes evidências genética e/ou inflamatória devido a sua associação com neuropatias periféricas sensoriais e motoras, como a síndrome de Charcot-Marie-Tooth, a ataxia de Friedreich e a neuropatia de Guillan Barrè. O acometimento auditivo, pode preceder em muitos anos o aparecimento da neuropatia periférica ${ }^{5}$. 
A neuropatia/dessincronia auditiva está relacionada com agenesia do nervo coclear, hiperbilirrubinemia, anóxia neonatal, esclerose múltipla, infecções congênitas como herpes zoster e citomegalovírus, ataxia de Friedrich e leucodistrofias, pois as mesmas podem gerar lesões no sistema nervoso central com preservação de células ciliadas externas e internas ${ }^{6}$.

As características audiológicas da neuropatia/dessincronia auditiva incluem: perda auditiva, geralmente bilateral, podendo ser de qualquer grau; funcionalidade das células ciliadas externas, evidenciada pela presença de emissões otoacústicas (EOA) e/ou microfonismo coclear; respostas anormais, nos potenciais evocados auditivos de tronco encefálico (PEATE); além do desempenho incompatível da percepção auditiva, baseado nos limiares tonais ${ }^{7}$. Os achados audiológicos são: presença de EOA e de microfonismo coclear, ausência de reflexos acústicos, ausência ou anormalidade dos PEATE, os limiares tonais podem estar próximos da normalidade ou perda auditiva de grau profundo, com discriminação de fala incompatível com o limiar para tom puro ${ }^{8}$.

Neto e colaboradores relataram 30 casos de neuropatia auditiva e observaram que o perfil da curva tonal, a inteligibilidade pobre e a possível incoerência da presença do reflexo do músculo do estribo, em conjunto ou separadamente, em paciente cuja queixa é "não entender a fala das pessoas", são sinais evidentes da presença de neuropatia auditiva ${ }^{9}$.

Alguns indivíduos com neuropatia/dessincronia auditiva podem apresentar limiares auditivos normais ou perda de graus variados, ausência de sincronia neural no potencial auditivo evocado de tronco encefálico, ausência de reflexos acústicos e presença de emissões otoacústicas com amplitudes exacerbadas, podendo haver ausência de supressão contralateral, ipsilateral ou biaural das EOA e ausência do limiar diferencial de mascaramento ${ }^{10}$.

É possível que algumas crianças apresentem perda de suas emissões otoacústicas evocadas com o passar do tempo, mas mantenham o registro do microfonismo coclear ${ }^{11}$.

Kós e Kós relatam que portadores de neuropatia/dessincronia auditiva diferenciam de outras alterações retrococleares, como neurinoma do acústico ou esclerose múltipla, por seus exames de imagens normais ${ }^{12}$.

Os recursos (re) habilitativos frente à neuropatia/dessincronia auditiva estendem-se da utilização de dispositivos de amplificação, o sistema de frequência modulada, o implante coclear até o implante de tronco encefálico. Todavia, estes podem não ser de acesso a todos ou não serem indicados ou insuficientes. Sob outra perspectiva, a terapia fonoaudiológica figura como um recurso disponível, indispensável e oportuna para a (re) habilitação auditiva.

Rondina e Matas relatam os encaminhamentos fonoaudiológicos que devem ser discutidos com a família e outros profissionais, para indivíduos com neuropatia/dessincronia auditiva como: terapia fonoaudiológica, protetização, implante coclear e implante de tronco encefálico ${ }^{13}$.

Resultados obtidos em crianças com neuropatia auditiva usuárias de implante coclear sugerem que a estimulação elétrica é capaz de compensar a dessincronia do nervo e que o implante coclear é um recurso para a (re) habilitação permitindo melhoria nas habilidades da audição e comunicação, mas as habilidades adquiridas parecem sofrer influência de fatores como tempo de uso do dispositivo, processo terapêutico sistemático, época do diagnóstico e a idade da criança ${ }^{14}$.

As estratégias de comunicação empregadas nas terapias fonoaudiológicas, podem ser recomendadas como forma de reabilitação auditiva amenizando a dificuldade de comunicação do indivíduo com neuropatia/dessincronia auditiva, pois esse recorre a alguns mecanismos para compreender o que está sendo falado e retornar a mensagem ao interlocutor com maior facilidade e segurança.

As estratégias de comunicação constituem um conjunto de determinadas atitudes que funcionam como agentes facilitadores para que a mensagem seja mais facilmente recebida visual ou auditivamente ${ }^{13}$. Boèchat, em 1992 identificou as estratégias de comunicação utilizada por deficientes auditivos e verificou sua funcionalidade. Também, organizou-as em grupos, classificou-as conforme sua natureza e concluiu que todas são úteis, mesmo aquelas que não colaboram tão eficazmente para a recepção da mensagem. Tais estratégias de comunicação podem ser divididas da seguinte maneira ${ }^{15}$ :

- Cognitivas: pressupõe o conhecimento sobre as características da deficiência auditiva. Sua aplicação conduz a mudança permanente no padrão de recepção da mensagem, dependem de um estudo prévio da situação para acertar a conduta e solucionar o que está dificultando a recepção da mensagem. Neste grupo, encaixam-se: leitura orofacial, contexto, atenção, organização, esclarecimento sobre a deficiência auditiva, questionamento, intuição e o ato de situar o interlocutor.

- Interventivas: atuação direta da deficiência auditiva quanto ao interlocutor e o ambiente. Pressupõe conhecimento das implicações do ruído, distância, iluminação, direcionalidade dos sons, entre outros, para a recepção da informação. 
São compostas por: aproximação ao falante, posicionamento favorável, afastamento do ruído, diminuição ou eliminação do ruído, iluminação adequada, solicitação de alteração de velocidade de fala, limitação do número de interlocutores, desobstrução da pista visual, posicionamento favorável do interlocutor e sugestão de local mais silencioso.

- Mecânicas: estratégias como manipulação do aparelho de amplificação sonora individual, utilização de dispositivos auxiliares e uso da ressonância natural com posicionamento da palma da mão ao pavilhão auricular.

- Paliativas: as estratégias dessa categoria não atingem a causa do problema, apenas geram redundância da mensagem. São elas: solicitação de repetição, solicitação de aumento ou diminuição da intensidade de voz e notificação de não entendimento.

- Remeativas: enquadram-se as estratégias, em que é percebido pelo deficiente auditivo que parte da mensagem já foi perdida e que há poucas chances de que consiga acompanhar a conversação sem problemas. Nessa categoria estão: solicitação de ajuda de terceiros, adiamento da conversação, evitar situação de comunicação e emprego da escrita.

- Desistivas: encontra as estratégias como isolamento da situação de comunicação e abandono social.

- Simulativas: estas estratégias são utilizadas para mascarar o não entendimento da mensagem. Os exemplos são: monopólio da conversação, entendimento simulado e distração simulada.

Os autores Horácio e Goffi-Gomez avaliaram a contribuição da leitura orofacial na percepção de fala em 4 indivíduos com neuropatia/dessincronia auditiva por meio de provas de percepção de fala sem e com leitura orofacial, que incluíram repetição de palavras dissilábicas e trissilábicas e repetição de frases em apresentação aberta (open-set). Constataram que o uso da leitura orofacial em provas específicas facilita a percepção auditiva e contribui para uma comunicação mais efetiva do neuropata auditivo ${ }^{16}$

Considerando que, as estratégias de comunicação figuram como uma alternativa para serem aproveitadas no processo (re) habilitativo, o objetivo foi investigar quais são as estratégias de comunicação utilizada por indivíduos que foram diagnosticados com neuropatia/dessincronia auditiva.

\section{MÉTODOS}

Este estudo foi realizado seguindo o padrão observacional transversal. No estudo os critérios estabelecidos para inclusão dos participantes foram: indivíduos adultos com diagnóstico audiológico de neuropatia/dessincronia auditiva, de ambos os sexos, na faixa etária de 18 a 60 anos, que realizam acompanhamento fonoaudiológico, usuários ou não de AASI. Como critério de exclusão os indivíduos não poderiam apresentam problemas como deficiência mental ou alteração de memória e também transtornos psiquiátricos.

Considerando os critérios estabelecidos a amostra foi composta por 10 indivíduos sendo seis do sexo masculino e quatro do sexo feminino, que atenderam aos critérios descritos. Em um primeiro momento, os participantes foram e esclarecidos sobre o objetivo pretendido com o estudo e a metodologia a ser aplicada, sendo que os mesmos concordaram em colaborar. Na sequência, houve a assinatura do termo de consentimento livre e esclarecido após concordância da participação.

Para verificar a utilização ou não das estratégias de comunicação e quais as de maior e menor ocorrência foi aplicado nos indivíduos portadores de neuropatia/dessincronia auditiva um questionário (Figura 1), o qual pontua as estratégias de comunicação segundo a classificação de Boèchat .

O estudo foi desenvolvido na Divisão de Saúde Auditiva do Hospital de Reabilitação de Anomalias Craniofaciais da Universidade de São Paulo, Campus Bauru, São Paulo mediante parecer favorável para execução pelo Comitê de Ética em Pesquisa com seres humanos sob número 286/2006.

Os resultados obtidos com os indivíduos que apresentam neuropatia/dessincronia auditiva serão apresentados de forma descritiva, por meio de tabela.

\section{RESULTADOS}

Os resultados revelam que dos 10 indivíduos adultos com diagnóstico audiológico de neuropatia/ dessincronia auditiva que participaram do estudo, todos utilizam estratégias de comunicação na tentativa de compensar o prejuízo auditivo gerado pela patologia, independente do uso de outros recursos para amenizar o problema de comunicação como, por exemplo, os dispositivos de amplificação.

Do total de participantes do estudo que apresentavam neuropatia/dessincronia auditiva, oito utilizavam aparelho de amplificação sonora individual como alternativa de compensar a deficiência auditiva, enquanto dois não utilizavam este dispositivo por julgarem que o mesmo não resolvia o problema 


\section{QUESTIONÁRIO}

1 - Quais as estratégias de comunicação você utiliza:

( ) Ênfase à leitura oro-facial

( ) Uso do contexto

( ) Uso de atenção

( ) Organização

( ) Esclarecimento sobre a deficiência auditiva

( ) Questionamento

( ) Intuição

( ) Manipulação do AASI

( ) Utilização de dispositivo auxiliar

( ) Uso ressonância natural

( ) Solicita ajuda de terceiros

2 - Quanto ao ambiente você procura:

( ) Afastar-se do ruído

( ) Diminuir o ruído

( ) Eliminar o ruído

( ) Procura iluminação melhor

3 - O que você solicita ao interlocutor quando não compreende a mensagem:

( ) Alterar velocidade

( ) Desobstrução pista visual

( ) Posicionamento favorável

( ) Solicita repetição da mensagem

( ) Aumento da intensidade da voz

( ) Diminuição intensidade da voz

( ) Notifica o não entendimento

( ) Aproximação ao falante

( ) Sugestão de local mais silencioso

4- O que você faz quando não compreende a mensagem:

( ) Adia a conversação

( ) Evita a situação de comunicação

( ) Utiliza a escrita

( ) Procura o isolamento

( ) Abandona a situação

( ) Mantém o monopólio da conversa

( ) Realiza entendimento simulado

( ) Realiza distração simulada

Figura 1 - Questionário de estratégias de comunicação

de comunicação. Como já mencionado, todos os indivíduos utilizaram as estratégias de comunicação, todavia, verificou-se que houve diminuição no seu emprego, quando o indivíduo está com o AASI, mas mesmo assim os usuários do dispositivo de amplificação não abandonam o uso dessa alternativa para facilitar a comunicação.
Também foi possível observar neste estudo que todas as estratégias de comunicação são empregadas por estes indivíduos.

Com relação à natureza das estratégias de comunicação as mais utilizadas em ordem de ocorrência foram: cognitiva, interventiva, mecânica, paliativa, remediativa, desistiva e, finalmente a simulativa. 
Estas estratégias foram referidas, por indivíduos usuários e não usuários de AASI conforme revelada na Tabela 1, a qual um apresenta os resultados da utilização das estratégias de comunicação quanto à natureza para os indivíduos avaliados usuários ou não usuários de AASI.

O gráfico 1 revela os resultados pontuando tipo de estratégia de comunicação de maior ocorrência segundo a natureza utilizada pelos indivíduos avaliados usuários e não usuários de AASI.

Quanto ao grupo das estratégias de comunicação de natureza cognitiva as de maior ocorrência foram: utilização de ênfase à leitura orofacial, atenção auditiva, utilização de questionamento, situar o interlocutor. Na estratégia interventiva, a procura de iluminação adequada foi à alternativa referida pela maioria dos participantes. Na mecânica, foi o uso de ressonância natural. A solicitação de repetição ocorreu com maior frequência na estratégia de comunicação de natureza paliativa. Na remediativa o adiamento da conversação e evitar a situação de comunicação. Já o isolamento foi o artifício aderido pelos participantes na maioria das vezes como parte da estratégia desistiva e o entendimento simulado ocorreram frequentemente para a estratégia simulativa.

É importante destacar que para os indivíduos não usuários de aparelho de amplificação sonora individual a estratégia de comunicação de natureza mecânica restringe-se à utilização da ressonância natural, ou seja, posicionamento da palma da mão de modo convexo no pavilhão auricular. Da mesma forma, a estratégia de comunicação de natureza simulativa apesar de adotada pelos indivíduos não aconteceu em todas as situações de comunicação, mas somente quando outras tentativas foram realizadas e não apresentaram sucesso.

Tabela 1 - Apresentação do número dos indivíduos quanto á utilização de estratégias de comunicação, segundo sua natureza

\begin{tabular}{|c|c|c|c|c|}
\hline \multirow{2}{*}{$\begin{array}{l}\text { Natureza da estratégia } \\
\text { de comunicação }\end{array}$} & \multicolumn{2}{|c|}{$\begin{array}{c}\text { Usuários de } \\
\text { AASI }\end{array}$} & \multicolumn{2}{|c|}{$\begin{array}{c}\text { Não usuários de } \\
\text { AASI }\end{array}$} \\
\hline & Sim & Não & Sim & Não \\
\hline Cognitiva & 7 & 1 & 2 & 0 \\
\hline Interventiva & 6 & 2 & 2 & 0 \\
\hline Mecânica & 7 & 1 & 1 & 1 \\
\hline Paliativa & 6 & 2 & 2 & 0 \\
\hline Remediativa & 3 & 5 & 0 & 2 \\
\hline Desistiva & 1 & 7 & 0 & 2 \\
\hline Simulativa & 4 & 4 & 1 & 1 \\
\hline
\end{tabular}

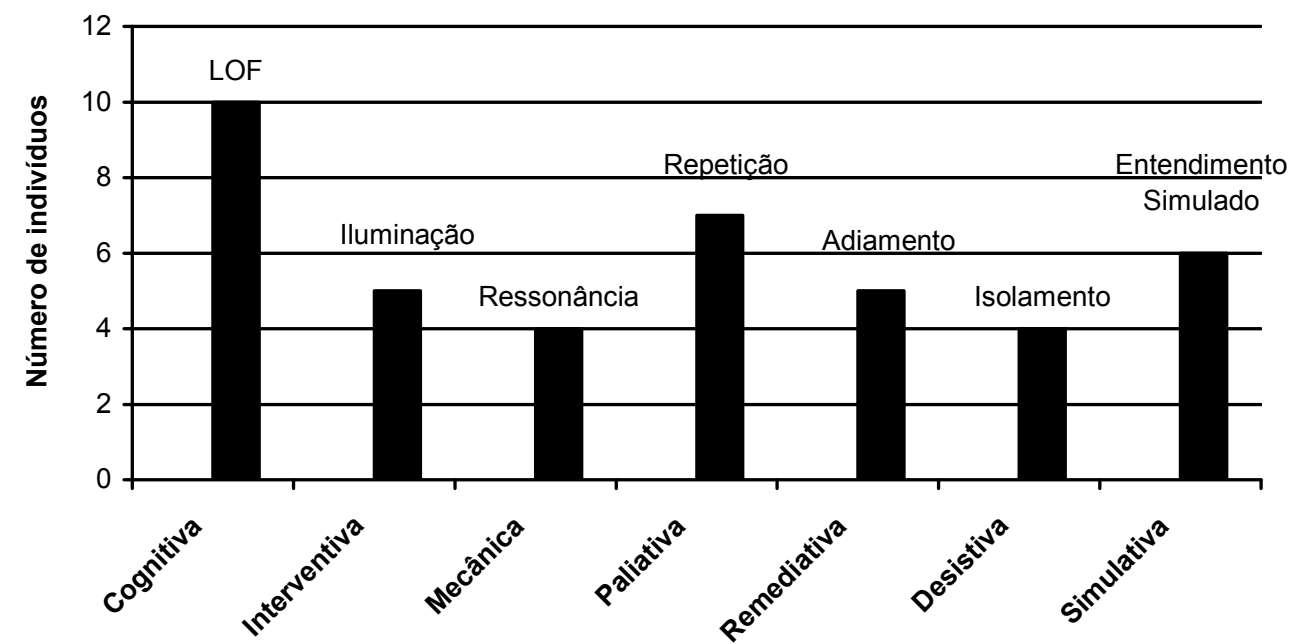

Gráfico 1 - Apresentação do tipo de estratégia de comunicação de maior ocorrência segundo a natureza utilizada pelos indivíduos avaliados 


\section{DISCUSSÃO}

Os recursos disponíveis para a reabilitação frente ao diagnóstico de neuropatia/dessincronia auditiva estendem-se da utilização de dispositivos eletrônicos, como o AASI, o sistema de frequência modulada, o implante coclear e atualmente 0 implante de tronco encefálico. Todavia, estes recursos podem não ser de acesso a todos os indivíduos ou aplicado a uma população específica. Em outro prisma, a terapia fonoaudiológica também figura como um recurso disponível para a reabilitação auditiva somando-se ou não a utilização dos recursos anteriormente citados sendo uma alternativa indispensável e oportuna.

Os autores Rondina e Matas indicam a terapia fonoaudiológica como recurso na tentativa de melhorar as habilidades linguísticas e auditivas para indivíduos diagnosticados com neuropatia/ dessincronia auditiva ${ }^{13}$. Desta forma, as estratégias de comunicação utilizadas nas terapias fonoaudiológicas, poderão ser recomendadas como forma de reabilitação amenizando a dificuldade de comunicação encontrada pelo indivíduo diagnosticado com neuropatia/dessincronia auditiva, pois este recorre a alguns mecanismos para compreender o que está sendo falado e retornar a mensagem ao interlocutor com maior facilidade e segurança como pode ser constatado pelos resultados apresentados nesse estudo.

Como já mencionado, todos os portadores de neuropatia/dessincronia auditiva utilizaram as estratégias de comunicação, mas houve diminuição no seu emprego, quando o indivíduo estava com o AASI. Com este resultado surge o questionamento quanto ao benefício proporcionado pelo AASI para a comunicação dos portadores de neuropatia/dessincronia auditiva, e, além disso, outra dúvida está em entender o porquê que a amplificação auxilia alguns indivíduos e outros não.

$\mathrm{Na}$ literatura os estudos que relacionam a neuropatia/dessincronia auditiva e estratégias de comunicação são escassos. No Brasil, somente os autores Horácio e Goffi-Gomez se propuseram a avaliar a contribuição da leitura orofacial na percepção de fala em indivíduos com neuropatia/dessincronia auditiva ${ }^{16}$.
Neste trabalho a leitura orofacial foi a estratégia de comunicação mais adotada pelos indivíduos, sendo que o mesmo resultado foi encontrado no estudo desenvolvido por Horácio e Goffi-Gomez ${ }^{16}$. Portanto, acredita-se que a leitura orofacial é uma ferramenta que pode ser utilizada com a maioria dos indivíduos com problemas de audição, bem como os com neuropatia auditiva,pois quanto maiores forem a informação auditiva e o contexto, com apoio na pista visual, melhor será o desempenho comunicativo.

Com este estudo observou-ser que as estratégias de natureza simulativa e desistiva são as últimas as serem empregadas para o problema de comunicação que acomete os indivíduos com neuropatia/dessincronia auditiva, mostrando que o indivíduo tenta de todas as maneiras participar do processo de comunicação efetivamente.

Os achados permitem incentivar a realização de novos trabalhos com o mesmo intuito, bem como a utilização de estratégias de comunicação que englobem o uso da leitura orofacial, principalmente para a melhora da percepção de fala.

Neste estudo, observou-se que as estratégias de comunicação foram adotadas espontaneamente por indivíduos portadores de neuropatia/dessincronia auditiva como tentativa de amenizar ou solucionar o déficit auditivo. Desta forma, é evidente que o emprego das estratégias de comunicação deve ser seguido pelos indivíduos com deficiência auditiva e difundido pelos profissionais como mais uma alternativa para se recorrer frente a este déficit sensorial.

\section{CONCLUSÃO}

Com os resultados obtidos pode-se concluir que todas as estratégias de comunicação das diversas naturezas são utilizadas por estes indivíduos com neuropatia/dessincronia auditiva. Portanto, esta alternativa terapêutica deve ser empregada e recomendada como forma de reabilitação auditiva, amenizando a dificuldade de comunicação dos indivíduos com diagnóstico de neuropatia/dessincronia auditiva. 


\section{ABSTRACT}

Purpose: to investigate which communication strategies, according to Boèchat (1992) classification, used by hearing impaired individuals with auditory neuropathy/dys-synchrony. Methods: A questionnaire prepared by Boèchat (1992) was applied, scoring the communication strategies. The questionnaire was used to check if the individuals with auditory neuropathy/dys-synchrony use or not communication strategies and to check their highest and lowest occurrence. Results: the results show that all participants use communication strategies as a way to reduce hearing loss problems, and that all strategies are used. Cognitive-nature communication strategies are the most used, while the desisting ones are the least used by people with auditory neuropathy/dys-synchrony, users or nonusers of some hearing device. Conclusion: the use of communication strategies is important for all individuals with auditory neuropathy/dys-synchrony as an alternative to make easier the communication.

KEYWORDS: Strategies; Hearing; Hearing Loss; Adults

\section{REFERÊNCIAS}

1. Martinho ACF. Neuropatia auditiva/dessincronia auditiva em crianças usuárias de implante coclear [tese] São Paulo (SP): Universidade de São Paulo; 2007.

2. Pearce W, Martin RL. On auditory neuropathy, aka auditory neuropathy spectrum. Nuts \& Bolts Hear J. 2009; 62(2):38-9.

3 .Zeng FG, Liu S. Speech perception in individuals with auditory neuropathy. J Speech Lang Hear Res.2006; 49(2): 367-80.

4. Melo TM, MORET ALM, BEVILACQUA MC. Avaliação da produção de fala em crianças deficientes auditivas usuárias de Implante Coclear Multicanal. Rev Soc Bras Fonoaudiol. 2008; 13(1): 45-51.

5. Fávero ML Romanos J, Mingroni-Netto RC, Balieiro CR, Donini TS, Spinelli M. Neuropatia auditiva decorrente de mutação no gene OTOF. Arq Int ORL. 2005; 9 (4): 25-30.

6. Neto OMS. Neuropatia auditiva. In: Lopes Filho O. Tratado de fonoaudiologia. 2. ed. Ribeirão Preto: Tecmedd; 2005. p. 301-15.

DOI: 10.1590/S1516-18462010005000053

RECEBIDO EM: 16/09/2009

ACEITO EM: 17/02/2010

Endereço para corresondência:

Jerusa Roberta Massola de Oliveira

Rua José Lúcio de Carvalho, 770

Jaú - SP

CEP: 17201-150

E-mail: jemassola@ hotmail.com
7. Parra VM, Matas CG. Estudo das características audiológicas em casos de neuropatia auditiva. PróFono. 2002; 14 (2): 241-6.

8. Beutner D, Foerst A, Lang-Roth R, von Wedel $\mathrm{H}$, Walger M Risk Factors for Auditory Neuropathy/ Auditory Synaptopathy. J for Oto Rhino Laringology Head and Neck Surgery .2007; 69(4): 239-44.

9. Neto OMS, Redondo MC, Carlos RC, Figueiredo MS, Lopes Filho OC. Neuropatia auditiva: aspectos relevantes na investigação clínica. Rev Bras Otorrinolaringol. 2001; 67 (5): 636-642.

10. Hood LJ, Berlin Cl. Auditory neuropathy (Auditory Dsy-synchrony) disables eferent supression of otoacoustic emissions. In: Sininger Y, Starr A. Auditory neuropathy: A new perspective on hearing disorders. 1. ed. San Diego: Singular; 2001. p.183-202.

11. Deltenre $P$, Mansbach AL, Bozet C, Barthelemy $P$, Paulissen D, Renglet T.. Auditory neropathy with preserved cochlear microphonics and secondary loss of otoacoustic emissions. Audiology. 1999; 38 (4): 187-95.

12. Kós AO, Kós MI. Etiologia das perdas auditivas e suas características audiológicas. In: Frota S. Fundamentos em fonoaudiologia: audiologia. 2. ed. Rio de Janeiro: Guanabara Koogan; 2003. p. 123-40.

13. Rondina C, Matas CG. Neuropatia auditiva: estudo de caso. Acta ORL. 2006; 24 (1): 10-7.

14. Silva RCL Araujo SG Os resultados do implante coclear em crianças portadoras de neuropatia auditiva: revisão de literatura Rev Soc Bras Fonoaudiol. 2007; 12(3): 252-7.

15. Boéchat EM. Ouvir sobre o prisma da estratégia. [dissertação]. São Paulo (SP): Pontifícia Universidade Católica; 1992.

16 - Horácio CP, Goffi-Gomez MVS. A contribuição da leitura orofacial na comunicação do neuropata auditivo. Rev. CEFAC 2007; 9 (3): 411-16. 